\title{
Avaliação de impacto da escala econômica na dimensão ambiental das empresas do ISE da BM\&FBOVESPA conforme parâmetros da Política Nacional do Meio Ambiente (Lei no 10.165)
}

\author{
Impact assessment of the economic scale in the environmental \\ dimension of the ISE companies of BM\&FBOVESPA as parameters of \\ the National Environmental Policy Act (Law No. 10,165)
}

\author{
Carlos Alberto Di Agustini ${ }^{1,2}$ \\ Cecília Maria Villas Boas de Almeida ${ }^{3}$ \\ Feni Dalano Roosevelt Agostinho ${ }^{3}$ \\ Biagio Fernando Giannetti ${ }^{3}$
}

\begin{abstract}
Resumo: Bolsa de valores é o espaço em que as empresas podem obter capital estimulando a atividade empreendedora. Os investidores ambientalmente responsáveis buscam investir em ações de empresas sustentáveis, até porque são mais rentáveis. Atendendo a essa demanda, foi criado no Brasil em 2005 o Índice de Sustentabilidade Empresarial (ISE) - iniciativa pioneira na América Latina e quarto indicador do tipo no mundo -, compara o desempenho das empresas listadas na Bolsa de Valores, Mercadorias e Futuros (BM\&FBOVESPA) sob os aspectos de um conceito de sustentabilidade. É um índice de ações referencial para os investimentos socialmente responsáveis, composto por empresas que se destacam em sustentabilidade a longo prazo. A finalidade desse índice é criar um ambiente de investimento compatível com as demandas de desenvolvimento sustentável da sociedade e estimular práticas mais sustentáveis nas empresas. Investimentos em empresas sustentáveis geram valor para o acionista, pois estão mais preparadas para enfrentar riscos econômicos, sociais e ambientais. Integrar o ISE é como ter um selo de qualidade reconhecido pelo mercado como empresa que atua com sustentabilidade. Para as 40 empresas integrantes do ISE em 2013, segundo parâmetros da Política Nacional do Meio Ambiente (Lei n 10.165 ), classifica-se a interferência da empresa no território pelo potencial de poluição e utilização de recursos naturais. Entretanto, a Lei $\mathrm{n}^{\circ} 10.165$ não contempla o impacto da escala econômica na classificação de potencial de poluição e utilização de recursos naturais pelas empresas. Ao considerar o impacto da escala econômica das empresas integrantes do ISE em 2013, amplia-se a classificação original da Lei $\mathrm{n}^{\circ} 10.165$, possibilitando que os stakeholders mensurem a interferência dessas empresas na biosfera. SABESP, CPFL ENERGIA, TELEFONICA BRASIL (Vivo), CEMIG, OI, BRF FOODS (Sadia, Perdigão, Batavo e Elegê), ELETROBRAS, BRASKEN, GERDAU, IPIRANGA e VALE são as empresas que exigem maior atenção por parte dos stakeholders porque possuem alto potencial de poluição e utilização de recursos naturais pelos parâmetros ampliados da Lei n ${ }^{\circ} 10.165$.
\end{abstract}

Palavras-chave: ISE da BM\&FBOVESPA. Potencial de poluição e utilização de recursos ambientais. Impacto pela escala econômica. Política Nacional do Meio Ambiente (Lei $n^{\circ}$ 10.165).

\begin{abstract}
Stock exchange is the space in which companies can raise capital by stimulating entrepreneurial activity. Environmentally responsible investors seek to invest in stocks of sustainable enterprises because they are more profitable. Given this demand, the Corporate Sustainability Index (ISE) was created in Brazil in 2005 - a pioneer initiative in Latin America and the fourth indicator of this kind in the world. This index compares the performance of the companies listed on the Securities, Commodities and Futures Exchange (BM\&FBOVESPA) considering aspects of the sustainability concept. It is an index of referential actions for socially responsible investments comprising companies that excel in sustainability in the long term. The purpose of this index is to create an environment for investment compatible with the demands of society's sustainable development and encourage more sustainable practices in businesses. Investments in sustainable companies create value for the shareholder, because these
\end{abstract}

\footnotetext{
Fundação Getulio Vargas - FGV. Praia de Botafogo, 190, $12^{\circ}$ andar, CEP 22.253-900, Rio de Janeiro, RJ, Brasil, e-mail: agustini@fgvmail.br

${ }^{2}$ Universidade Municipal de Ensino Superior de São Caetano do Sul - USCS, Av. Goiás, 3.400, CEP 09550-051, São Caetano do Sul, SP, Brasil
}

Laboratório de Produção e Meio Ambiente, Programa de Pós-Graduação em Engenharia de Produção, Universidade Paulista - UNIP, Rua Dr. Bacelar, 1212, CEP 04026-002, São Paulo, SP, Brasil, e-mail: cmvbag@unip.br; Feni@unip.br; biafgian@unip.br 
companies are better prepared to face economic, social and environmental risks. Integrating the ISE is equivalent to having a quality seal recognized by the market as a company that operates under the concepts of sustainability. For the 40 companies listed in the ISE in 2013, according to standards of the National Environmental Policy Act (Law No. 10,165), interference on a territory was ranked by their potential for pollution and use of natural resources. However, Law No. 10,165 does not include in its classification the economic scale impact of the potential pollution and use of natural resources of these enterprises. When the impact of the economic scale of the members of the ISE in 2013 is considered, the original classification of Law No. 10,165 is expanded, enabling stakeholders to measure the interference of these companies in the biosphere. SABESP, CPFL ENERGIA, TELEFONICA BRASIL (Vivo), CEMIG, OI, FOODS BRF (Sadia, Perdigão, Batavo and Elegê), ELETROBRAS, BRASKEN, GERDAU, IPIRANGA and VALE are companies that require greater attention from stakeholders, because they present high potential for pollution and use of natural resources according to the extended parameters of Law No. 10,165.

Keywords: ISE BM\&FBOVESPA. Potential pollution and use of environmental resources. Impact of the economic scale. The National Environmental Policy Act (Law No. 10,165).

\section{Introdução}

Segundo Giannetti (apud DI AGUSTINI, 2009), a bolsa de valores é o espaço em que as empresas podem obter recursos estimulando a atividade empreendedora. A Figura 1 apresenta os principais componentes do sistema de investimento por meio de uma bolsa de valores.

Os principais fluxos de um sistema produtivo simples estão apresentados na Figura 1. Representação dos bens e trabalho (B e T) como uma fonte (simbolizado pelo círculo) que fornece recursos para a empresa (linha contínua). O fluxo do produto Y, consumido no mercado, é representado por uma linha contínua. A linha pontilhada representa os fluxos de dinheiro que são empregados nas transações (rombo) de venda do produto Y e de compra dos recursos B e T. O estoque D de capital é formado pelo capital

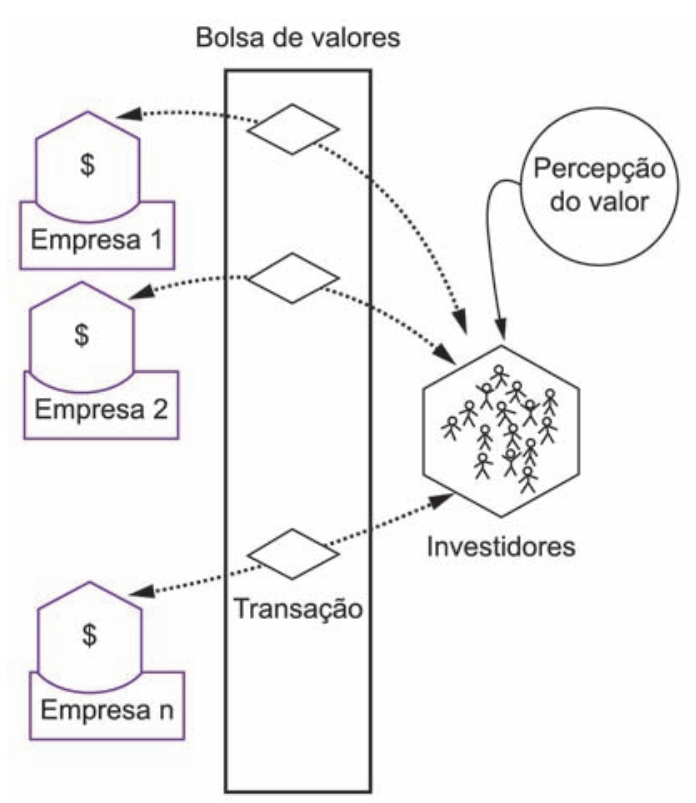

Figura 1. Representação dos fluxos de capital pela bolsa de valores. Fonte: GIANNETTI (apud DI AGUSTINI, 2009, p. 16). inicial somado à diferença do capital adquirido nas vendas do produto $\mathrm{Y}$ pelo capital empregado na compra de B e T.

Outro aspecto que pode ser observado na Figura 1 é que os fluxos de recursos e produtos são paralelos aos fluxos de dinheiro com sentido oposto. Os dois fluxos são interdependentes: sem recursos e produtos não é possível promover o fluxo de dinheiro e, por sua vez, sem capital não ocorrem os fluxos de recursos e de produtos. A transação ocorre sob controle do preço que é resultado do valor de mercado, obedecendo a condições ideais ao princípio econômico do equilíbrio entre a oferta e a demanda.

$\mathrm{O}$ valor de mercado, definido como o que as pessoas estão dispostas a pagar, é o principal método de contabilização usado em economia. Este valor é determinado pela percepção humana das necessidades em curto prazo e dos benefícios esperados. Por este motivo, o preço é representado na Figura 1 como um controle externo da transação.

Para aprofundar a discussão sobre esse tema, a Figura 2 amplia o diagrama da Figura 1. Fluxos e componentes importantes na sustentabilidade do sistema estão destacados, na Figura 2, com linhas mais grossas (GIANNETTI, apud DI AGUSTINI, 2009).

A Figura 2 mostra os principais componentes do sistema de investimento intermediado pela bolsa de valores. Os investidores podem realizar as transações na bolsa de valores, por meio de corretoras, individualmente, participando de clubes de investimento ou adquirindo fundos de investimento (via corretoras, banco ou gestor de recursos independente autorizado pela CVM).

Segundo Giannetti (apud DI AGUSTINI, 2009), os fluxos dos recursos e serviços da natureza são imprescindíveis para o funcionamento do sistema econômico e social. Na Figura 2, os recursos ambientais são diferenciados em renováveis $(\mathrm{R})$ e não renováveis $(\mathrm{N})$. Outro fato a destacar é que os recursos naturais não são acompanhados pelo fluxo de dinheiro. 
O dinheiro somente circula na parte direita da Figura 3, sendo empregado nas transações por recursos fornecidos pelo mercado, pelos serviços e produtos (bens ou serviços) gerados. Não é empregado dinheiro na produção ambiental, que fornece água limpa, solo fértil, boas condições climáticas, entre outros, para o suporte da vida na sociedade e para a economia. Estes recursos gratuitos são produzidos no sistema ambiental, que faz parte do sistema de sobrevivência com o de produção de alimentos.

Como o dinheiro é empregado para pagar as pessoas e nunca para recompensar o meio ambiente por sua contribuição, o valor de mercado não pode ser empregado para determinar a real riqueza recebida do meio ambiente. Quando os recursos ambientais são abundantes, pouco trabalho é necessário para sua exploração, os custos são baixos e os preços subestimam a real contribuição para o sistema econômico. Por outro lado, recursos escassos terão no sistema econômico preços elevados. Preços de mercado não são proporcionais à contribuição dada à economia pelos recursos do meio ambiente

No processo de busca do valor sustentável das empresas pelos investidores, a natureza do crescimento econômico é fundamental para compreender melhor a relação entre crescimento econômico e investimento, em que (Equação 1):

$$
\mathrm{D} \rightarrow \mathrm{A} \rightarrow \mathrm{D}^{\prime}
$$

D é o dinheiro, A é uma atividade econômica e $\rightarrow$ é o símbolo do fluxo de dinheiro. O investimento é feito com a expectativa que D' > D, ou seja, a obtenção de mais moeda. Para um empreendedor, o interesse não é o acúmulo de produtos (bens ou serviços), mas o de dinheiro.

A atividade A pode ser representada por (Equação 2):

$$
(\mathrm{B}, \mathrm{T}) \Rightarrow \mathrm{Y}
$$

sendo $\mathrm{B}$ os bens instrumentais, $\mathrm{T}$ o trabalho necessário para que ocorra o processo, resultando no produto (bem ou serviço) Y e o símbolo $\Rightarrow$ representa o processo.

Dessa forma, o sistema de produção é representado pela Equação 3:

$$
\mathrm{D} \rightarrow(\mathrm{B}, \mathrm{T}) \Rightarrow \mathrm{Y} \rightarrow \mathrm{D}^{\prime}
$$

tendo como expectativa pelos investidores que D' $>$ D.

Do ponto de vista do agente empreendedor, o capital investido D possibilita adquirir e organizar os bens instrumentais (B) e o trabalho (T). A intensidade do investimento está fundamentada na expectativa da venda futura dos bens ou serviços que irão ser produzidos. A expectativa do empreendedor baseia-se nos planos da empresa que são muito influenciados pelas condições econômicas do mercado.

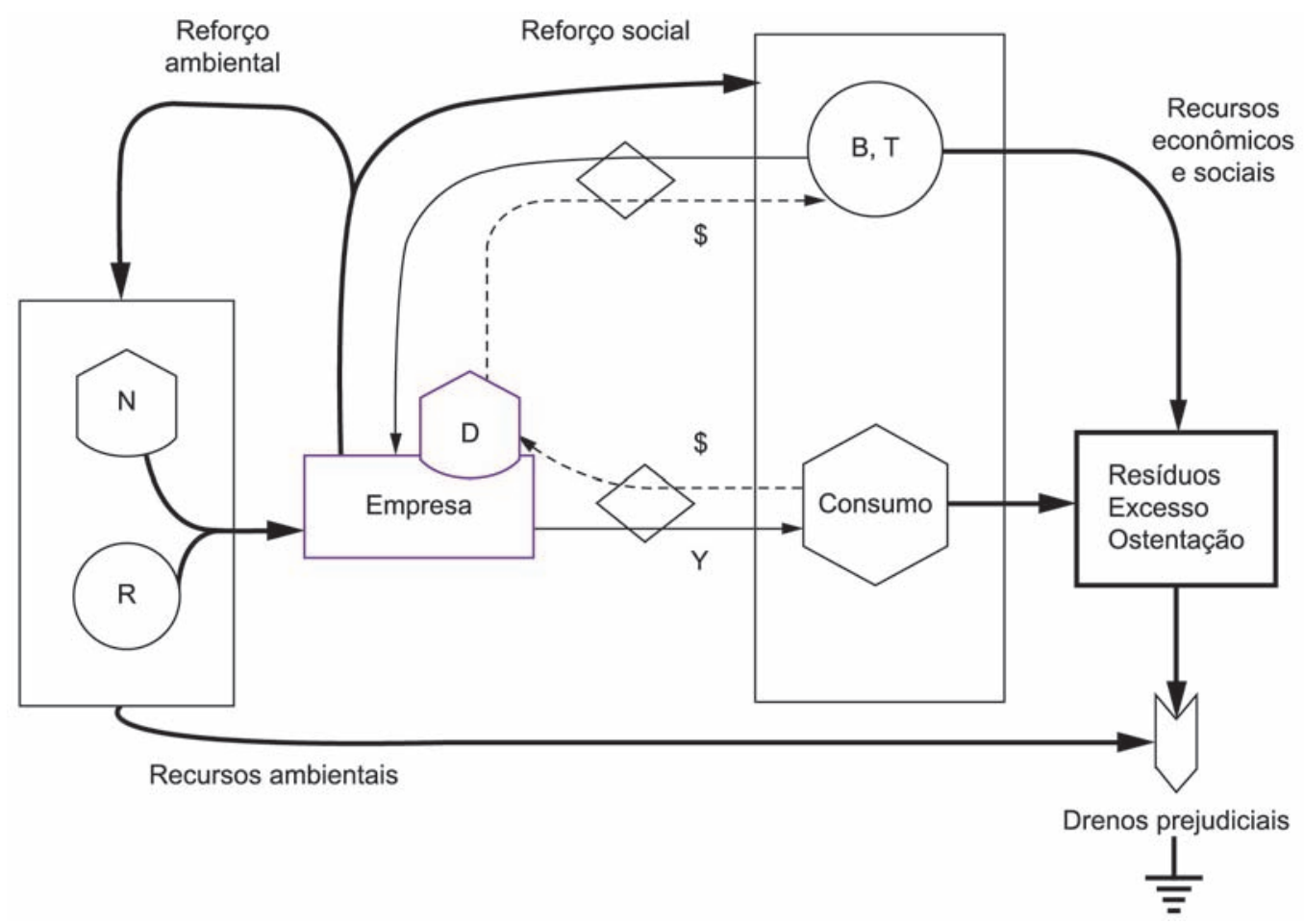

Figura 2. Fluxos e componentes importantes na valoração do investimento socialmente responsável. Fonte: GIANNETTI (apud DI AGUSTINI, 2009, p. 19). 


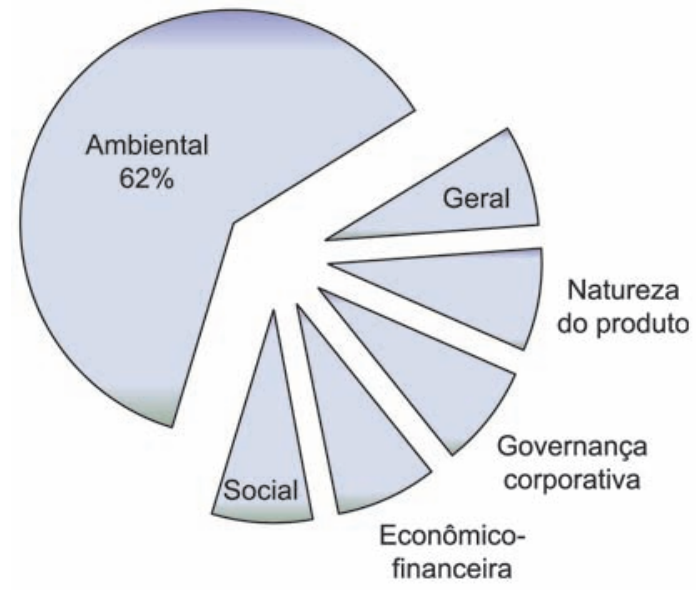

Figura 3. Desempenho do ISE e do Ibovespa.

O dinheiro somente circula na parte direita da Figura 2, sendo empregado nas transações por recursos fornecidos pelo mercado, pelos serviços e produtos (bens ou serviços) gerados. Não é empregado dinheiro na produção ambiental, que fornece recursos e serviços gratuitos, como água limpa, solo fértil, boas condições climáticas, entre outros, para o suporte da vida na sociedade e para a economia. Esses recursos gratuitos são produzidos no sistema ambiental, que faz parte do sistema social, econômico e empresarial.

Como o dinheiro é empregado para pagar as pessoas e nunca para recompensar o meio ambiente por sua contribuição, o valor de mercado não pode ser empregado para determinar a real riqueza recebida do meio ambiente. Quando os recursos ambientais são abundantes, pouco trabalho é necessário para sua exploração, os custos são baixos e os preços subestimam a real contribuição para o sistema econômico. Por outro lado, recursos escassos terão no sistema econômico preços elevados. Preços de mercado não são proporcionais à contribuição dada à economia pelos recursos do meio ambiente. Outro aspecto importante para o investidor socialmente responsável, representado na Figura 2, são os drenos prejudiciais à sustentabilidade.

Entre as diferentes escolhas de investimento, a percepção do valor das ações e das empresas é muito importante para a tomada de decisão do investidor, influenciada por fatores como liquidez, retorno e risco, mensurados com informações objetivas e subjetivas, provenientes da bolsa de valores e de outras fontes. Essa percepção de valor pelo mercado é também influenciada por questões de natureza ambiental (maior uso de recursos R, minimização de uso de recursos $\mathrm{N}$, gestão de drenos prejudiciais à sustentabilidade), podendo interferir na rentabilidade e na sobrevivência das empresas (BERTOLINI; ROJO; LEZANA, 2012).

De acordo com Shirreff (2004), a bolsa de valores surgiu de um pântano que era a Holanda há mil anos. Os holandeses aprenderam a domar a natureza, construíram represas, canais e moinhos para bombear águas. Os nomes das grandes cidades holandesas ecoam esse passado: dam significa represa, daí Amsterdam e Roterdam. Até os sobrenomes das pessoas: Van Damme, da represa; Van Dijck do dique.

Antes do Renascimento, a Holanda já era uma república e controlava um volume de comércio maior do que o da Inglaterra e França juntas. As especiarias asiáticas já eram apreciadas pelos europeus. Os indianos enchiam barcos com as especiarias e velejavam até a Península Arábica para vender. De lá, os temperos eram transportados em lombos de camelos até o Egito, onde eram revendidos para intermediários por Alexandria. Os venezianos tinham firmado um contrato exclusivo com os egípcios para revender as especiarias - inspiração para Shakespeare em O Mercador de Veneza (FERREIRA, s/d).

Dom João II, Rei de Portugal, decidiu investir na busca de especiarias direto na Índia para ganhar dinheiro e baratear o preço. Quando uma expedição saia à Índia, cerca de metade das caravelas conseguia voltar. Em 1502, Vasco da Gama partiria pela segunda vez à Índia com 20 navios fortemente armados. Voltou a Portugal em 1503 com 13 navios e 1.700 toneladas de especiarias - praticamente a mesma quantidade que Veneza importava do oriente por ano (FERNANDÉS-ARMESTO, 2009).

$\mathrm{Na}$ época, os holandeses também eram bons navegadores e como o risco das expedições era muito alto, o governo holandês uniu seis Companhias das Índias, formando uma grande estatal - a primeira grande corporação da história: Vereennigde Nederlandssche Oostindishe Compagnie. Dividiram o capital da empresa em pedaços (ações) para vender à população que era convidada a ser investidora e sócia. Um local para vender as ações foi construído e recebeu o nome de bourse (bolsa). Muitos investidores se interessavam pela nova atração, até um hotel foi construído em frente ao local da bourse - Hôtel des Bourses. O nome do título mobiliário ação, que representa uma fração do patrimônio líquido da empresa, vem do termo empreitada de alto risco, já que o dinheiro era destinado a financiar difíceis e arriscadas viagens à Índia para comprar especiarias. Se os navios voltassem das viagens com especiarias, os investidores receberiam uma parte dos lucros do negócio (dividendos).

$\mathrm{Na}$ época, a Espanha estava em guerra com a Holanda e os ataques e saques a navios não eram raros - tanto que os navios mercantes eram fortemente 
armados com canhões. Boatos que os navios estavam voltando carregados com especiarias - sinal de bastante lucro, o preço das ações subia na bourse. Mas, quando surgia informação de que os navios tinham sido atacados e afundados, o preço das ações caia na bourse.

O complexo funcionamento das bolsas de valores, atualmente ainda segue princípios das flutuações de preços da antiga bourse, e incorpora também percepções de valor dos investidores, dentre eles, questões relativas à interferência das empresas no meio ambiente e respectivos impactos ambientais, discutidos principalmente sob a égide da sustentabilidade.

Para Campos et al. (2013), a partir de um longo processo histórico de amadurecimento da consciência humana diante do desenvolvimento econômico, a gestão ambiental passou a incorporar diversas iniciativas governamentais e empresariais que procuraram formas de gestão mais adequadas para garantir um futuro melhor para o planeta. Nessa direção, a Lei $\mathrm{n}^{\circ} 10.165$, de 27 de dezembro de 2000, que dispõe sobre a Política Nacional do Meio Ambiente, é um marco regulatório legal sobre os impactos provocados pela interferência das empresas no meio ambiente (BRASIL, 2000).

O objetivo deste trabalho é realizar uma avaliação de impacto da escala econômica das empresas integrantes do ISE em conformidade com os parâmetros da Política Nacional do Meio Ambiente (Lei $\mathrm{n}^{\circ} 10.165$ ), que considera o potencial de poluição e utilização de recursos naturais.

\section{Definições}

Os impactos ambientais, a partir da Revolução Industrial ocorrida há aproximadamente 250 anos, provocaram ações e iniciativas envolvendo vários segmentos da sociedade com o objetivo de conscientizar a população e mudar a postura dos indivíduos e das empresas. São inúmeras as ações e iniciativas, dentre elas: Conferência da Biosfera (Paris, 1968); Conferência das Nações Unidas sobre Meio Ambiente (Estocolmo, 1972); Eco 92 ou Rio 92 (Rio de Janeiro, 1992); Cúpula Mundial para o Desenvolvimento Sustentável ou Rio +10 (Johanesburgo, 2002); Painel Intergovernamental sobre Mudanças Climáticas (Paris, 2007); e Relato Integrado promovido pelo International Integrated Reporting Council (IIRC), organização que promove a integração entre as informações financeiras, de sustentabilidade e de governança em relatórios corporativos, lançado mundialmente em 16 de abril de 2013, no Brasil, realizado pela BM\&FBOVESPA (ALVES; BARBOSA, 2013).

\subsection{ISE da BM\&FBOVESPA}

Para atender à demanda de investidores que buscam investir em ações e títulos de empresas comprometidas com práticas de gestão sustentáveis, foi criado em 2005 no Brasil o Índice de Sustentabilidade Empresarial (ISE), quarto indicador no mundo e iniciativa pioneira na América Latina. É uma ferramenta para análise comparativa do desempenho das empresas listadas na Bolsa de Valores, Mercadorias e Futuros (BM\&FBOVESPA), cuja finalidade é criar um ambiente de investimento compatível com as demandas de desenvolvimento sustentável da sociedade e estimular práticas mais sustentáveis nas empresas (BASSETTO, 2010).

O Conselho Deliberativo do ISE é composto por membros das seguintes instituições: ABRAPP; ANBID; APIMEC; BM\&FBOVESPA; ETHOS; IBGC; IFC; MMA e PNUMA. A Fundação Getulio Vargas (FGV) é a instituição responsável pela pesquisa e metodologia de listagem das empresas. Em 2013, o ISE era composto por 40 empresas participantes, conforme apresentado na Tabela 1 .

Investimentos em empresas sustentáveis geram valor para o acionista, a longo prazo, pois estão mais preparadas para enfrentar riscos econômicos, sociais e ambientais. Integrar o ISE é como ter um selo de qualidade reconhecido pelo mercado como empresa que atua com sustentabilidade. É o quarto indicador do tipo no mundo que compara o desempenho de empresas listadas na BM\&FBOVESPA sob os aspectos da sustentabilidade (MARCONDES; BACARJI, 2010).

O conceito de sustentabilidade empresarial adotado pelo ISE envolve várias dimensões. A dimensão ambiental se destaca pela sua relevância porque representa $62 \%$ do total de pesos para inclusão de uma empresa no índice, conforme Figura 4.

A BM\&FBOVESPA atribui peso 100 para cada uma das dimensões do questionário. Cada dimensão é subdivida em critérios, e as ponderações desses critérios são definidas pela relevância do tema no contexto atual da gestão empresarial e das demandas da sociedade (ISE METODOLOGIA, 2012).

$\mathrm{Na}$ dimensão ambiental, os pesos dos critérios são apresentados na Tabela 2, em que é atribuído maior grau de ponderação para o requisito gestão das empresas.

Desde a sua criação, em dezembro de 2005 , até 2013, o ISE apresentou taxa de crescimento acumulado superior ao do Ibovespa, conforme Tabela 3.

Apesar de o desempenho do ISE ser superior ao desempenho do Ibovespa no período de 2006 a 2012, até 2010 o Ibovespa apresentava desempenho superior ao ISE (Figura 3). 
A carteira do ISE é revisada anualmente e sua formação é feita por empresas pré-selecionadas, que respondem os questionários, e o Conselho Deliberativo escolhe aquelas com melhor classificação. A Tabela 4 apresenta as várias dimensões e respectiva quantidade de questões dos questionários enviados às empresas, no período de 2005 até 2013.

Tabela 1. Classificação da dimensão ambiental das empresas pelo impacto da escala econômica.

\begin{tabular}{|c|c|c|c|c|c|}
\hline & $\begin{array}{c}\text { Empresa do ISE em } \\
2013\end{array}$ & $\begin{array}{l}\text { Escala econômica } \\
\text { (R\$) em } 2012\end{array}$ & $\begin{array}{c}\text { Lei no } 10.165(\mathrm{Pp} / \\
\text { urn*) - classificação } \\
\text { original }\end{array}$ & $\begin{array}{c}\text { Lei }^{0} \\
10.165 \text { - classificação } \\
\text { de impacto }\end{array}$ & $\begin{array}{l}\text { Posição } \\
\text { relativa }\end{array}$ \\
\hline 1 & BRADESCO & 78.684 .684 .000 & $\mathrm{~S} / \mathrm{c}$ & $\mathrm{S} / \mathrm{c}$ & $\mathrm{S} / \mathrm{c}$ \\
\hline 2 & BICBANCO & 2.070 .723 .000 & $\mathrm{~S} / \mathrm{c}$ & $\mathrm{S} / \mathrm{c}$ & $\mathrm{S} / \mathrm{c}$ \\
\hline 3 & ITAUUNIBANCO $* *$ & 99.878 .400 .000 & $\mathrm{~S} / \mathrm{c}$ & $\mathrm{S} / \mathrm{c}$ & $\mathrm{S} / \mathrm{c}$ \\
\hline 4 & SANTANDER BR & 55.829 .354 .000 & $\mathrm{~S} / \mathrm{c}$ & $\mathrm{S} / \mathrm{c}$ & $\mathrm{S} / \mathrm{c}$ \\
\hline 5 & SUL AMERICA & 10.616 .700 .000 & $\mathrm{~S} / \mathrm{c}$ & $\mathrm{S} / \mathrm{c}$ & $\mathrm{S} / \mathrm{c}$ \\
\hline 6 & BANCO DO BRASIL & 69.355 .842 .000 & $\mathrm{~S} / \mathrm{c}$ & $\mathrm{S} / \mathrm{c}$ & $\mathrm{S} / \mathrm{c}$ \\
\hline 7 & ULTRACARGO**** & 301.000 .000 & Alto & 2.709 .000 .000 & $\mathrm{~B} \downarrow$ \\
\hline 8 & EVEN & 2.162 .240 .000 & Baixo & 6.486 .720 .000 & $\mathrm{~B}\rceil$ \\
\hline 9 & ECORODOVIAS & 2.409.107.000 & Baixo & 7.227.321.000 & $\mathrm{B} \downarrow$ \\
\hline 10 & ELEKEIROZ*** & 899.809 .000 & Alto & 8.098 .281 .000 & $\mathrm{~B} \downarrow$ \\
\hline 11 & ITAUTEC ** & 1.545 .300 .000 & Médio & 9.271 .800 .000 & $\mathrm{~B} \rightarrow$ \\
\hline 12 & AES TIETE & 2.112 .435 .000 & Médio & 12.674 .610 .000 & $\mathrm{~B} \rightarrow$ \\
\hline 13 & CCR & 5.767 .698 .000 & Baixo & 17.303.094.000 & $\mathrm{B} \rightarrow$ \\
\hline 14 & COELCE & 2.893 .720 .000 & Médio & 17.362 .320 .000 & $\mathrm{~B} \uparrow$ \\
\hline 15 & CESP & 3.354 .005 .000 & Médio & 20.124 .030 .000 & $\mathrm{~B} \uparrow$ \\
\hline 16 & COPASA & 3.519 .027 .000 & Médio & 21.114 .162 .000 & $\mathrm{~B} \uparrow$ \\
\hline 17 & OXITENO**** & 2.929 .000 .000 & Alto & 26.361 .000 .000 & $\mathrm{~B} \uparrow$ \\
\hline 18 & TRACTEBEL & 4.912 .499 .000 & Médio & 29.474 .994 .000 & $\mathrm{M} \downarrow$ \\
\hline 19 & DURATEX $^{* *}$ & 3.394 .399 .000 & Alto & 30.549 .591 .000 & $\mathrm{M} \downarrow$ \\
\hline 20 & ULTRAGAZ $* * *$ & 3.847 .000 .000 & Alto & 34.623 .000 .000 & $\mathrm{M} \downarrow$ \\
\hline 21 & WEG & 5.972 .118 .000 & Médio & 35.832 .708 .000 & $\mathrm{M} \downarrow$ \\
\hline 22 & ENERGIAS BR & 6.567 .300 .000 & Médio & 39.403 .800 .000 & $\mathrm{M} \rightarrow$ \\
\hline 23 & LIGHT & 7.613.096.000 & Médio & 45.678 .576 .000 & $\mathrm{M} \rightarrow$ \\
\hline 24 & SUZANO PAPEL & 5.192 .292 .000 & Alto & 46.730 .628 .000 & $\mathrm{M} \rightarrow$ \\
\hline 25 & COPEL & 8.532 .200 .000 & Médio & 51.193 .200 .000 & $\mathrm{M} \uparrow$ \\
\hline 26 & FIBRIA & 6.174 .373 .000 & Alto & 55.569 .357 .000 & $\mathrm{M} \uparrow$ \\
\hline 27 & TIM & 18.763 .947 .000 & Médio & 56.291 .841 .000 & $\mathrm{M} \uparrow$ \\
\hline 28 & NATURA & 6.345 .669 .000 & Alto & 57.111 .021 .000 & $\mathrm{M} \uparrow$ \\
\hline 29 & ELETROPAULO & 9.958 .198 .000 & Médio & 59.749 .188 .000 & $\mathrm{M} \uparrow$ \\
\hline 30 & SABESP & 10.754 .435 .000 & Médio & 64.526 .610 .000 & $\mathrm{~A} \downarrow$ \\
\hline 31 & CPFL ENERGIA & 15.055 .147 .000 & Médio & 90.330 .882 .000 & $\mathrm{~A} \downarrow$ \\
\hline 32 & TELEF BRASIL & 33.931 .422 .000 & Médio & 101.794 .266 .000 & $\mathrm{~A} \downarrow$ \\
\hline 33 & CEMIG & 18.460 .000 .000 & Médio & 110.760 .000 .000 & $\mathrm{~A} \rightarrow$ \\
\hline 34 & OI & 25.169 .230 .000 & Médio & 151.015 .380 .000 & $\mathrm{~A} \rightarrow$ \\
\hline 35 & BRF FOODS & 28.517 .000 .000 & Médio & 171.102 .000 .000 & $\mathrm{~A} \rightarrow$ \\
\hline 36 & ELETROBRAS & 34.064 .477 .000 & Médio & 204.386 .862 .000 & $\mathrm{~A} \rightarrow$ \\
\hline 37 & BRASKEM & 35.513 .397 .000 & Alto & 319.620 .573 .000 & $\mathrm{~A} \uparrow$ \\
\hline 38 & GERDAU & 37.981 .668 .000 & Alto & 341.835 .012 .000 & $\mathrm{~A} \uparrow$ \\
\hline 39 & IPIRANGA $* * *$ & 46.833 .000 .000 & Alto & 421.497 .000 .000 & $\mathrm{~A} \uparrow$ \\
\hline 40 & VALE & 93.511 .000 .000 & Alto & 841.599 .000 .000 & $\mathrm{~A} \uparrow$ \\
\hline
\end{tabular}

S/c: sem classificação segundo a Lei ${ }^{\circ} 10.165$; *Potencial de poluição e utilização de recursos naturais; **Empresas integrantes do grupo ITAUSA (ITSA3 e ITSA4); ***Empresas integrantes do grupo ULTRAPAR (UGPA3). Fonte: BM\&FBOVESPA (2013a). 


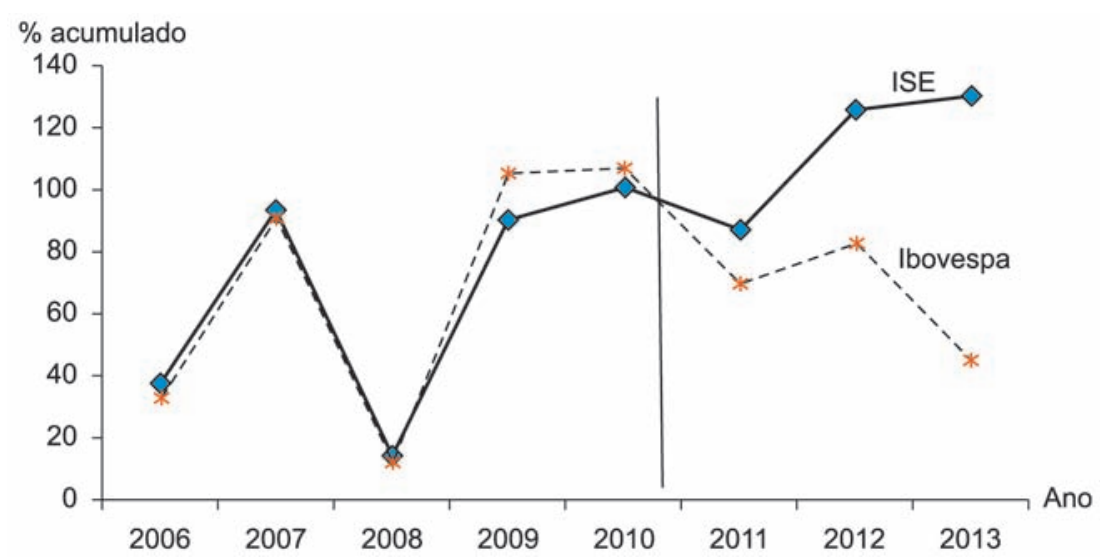

Figura 4. Proporção da dimensão ambiental do ISE. Fonte: ISE METODOLOGIA (2013).

Tabela 2. Percentual dos pesos da dimensão ambiental.

\begin{tabular}{lc}
\hline \multicolumn{1}{c}{ Critério } & Peso \\
\hline Política & $10,83 \%$ \\
Gestão & $40,83 \%$ \\
Desempenho & $31,67 \%$ \\
Cumprimento legal & $16,67 \%$ \\
\hline
\end{tabular}

Tabela 3. Taxas de crescimento (\%) do ISE e do Ibovespa.

\begin{tabular}{ccc}
\hline Ano & ISE & Ibovespa \\
\hline 2006 & $+37,8$ & $+32,9$ \\
2007 & $+40,4$ & $+43,6$ \\
2008 & $-41,1$ & $-41,2$ \\
2009 & $+66,4$ & $+82,6$ \\
2010 & $+5,8$ & $+1,0$ \\
2011 & $-6,7$ & $-17,9$ \\
2012 & $+20,5$ & $+7,4$ \\
2013 & $+1,94$ & $-20,64$ \\
Acumulado & $+129,9$ & $+44,82$ \\
Média anual & $+10,97$ & $+4,74$ \\
Média mensal & $+0,87$ & $+0,39$ \\
\hline
\end{tabular}

Fonte: BM\&FBOVESPA (2014).

A área demarcada na Tabela 4 mostra a relevância da dimensão ambiental pela quantidade de questões do ISE de 2005 até 2013, nela a representação em quantidade de questões passou de $35 \%$ em 2005 para $54 \%$ em 2013.

\subsection{Lei no $\mathbf{1 0 . 1 6 5 ~ - ~ P o l i ́ t i c a ~ N a c i o n a l ~ d o ~}$ Meio Ambiente}

Silva e Quelhas (2006) defendem que o governo exerce um papel relevante nas questões que envolvem interferência das empresas no meio ambiente, principalmente definindo a estrutura legal e regulatória com o objetivo de prover o desenvolvimento econômico preservando a qualidade de vida no ambiente.

A Lei $\mathrm{n}^{\circ}$ 10.165, de 27 de dezembro de 2000, dispõe sobre a Política Nacional do Meio Ambiente, assim como seus fins e mecanismos de formulação e aplicação (BRASIL, 2000). A Política Nacional do Meio Ambiente tem por objetivo a preservação, melhoria e recuperação da qualidade ambiental propícia à vida, visando assegurar, no País, condições ao desenvolvimento socioeconômico, aos interesses da segurança nacional e à proteção da dignidade da vida humana, atendidos os seguintes princípios:

- ação governamental na manutenção do equilíbrio ecológico, considerando o meio ambiente como um patrimônio público a ser necessariamente assegurado e protegido, tendo em vista o uso coletivo;

- racionalização do uso do solo, do subsolo, da água e do ar;

- planejamento e fiscalização do uso dos recursos ambientais;

- proteção dos ecossistemas, com a preservação de áreas representativas;

- controle e zoneamento das atividades potencial ou efetivamente poluidoras;

- incentivos ao estudo e à pesquisa de tecnologias orientadas para o uso racional e a proteção dos recursos ambientais;

- acompanhamento do estado da qualidade ambiental;

- recuperação de áreas degradadas;

- proteção de áreas ameaçadas de degradação; e

- educação ambiental a todos os níveis de ensino, inclusive a educação da comunidade, objetivando capacitá-la para participação ativa na defesa do meio ambiente.

Algumas definições são previstas na Lei ${ }^{\circ} 10.165$ (BRASIL, 2000): 
Tabela 4. Quantidade de questões do ISE.

\begin{tabular}{lcccccccc}
\hline \multicolumn{1}{c}{ Dimensão } & $\mathbf{2 0 0 5 / 6}$ & $\mathbf{2 0 0 6 / 7}$ & $\mathbf{2 0 0 7 / 8}$ & $\mathbf{2 0 0 8 / 9}$ & $\mathbf{2 0 0 9 / 1 0}$ & $\mathbf{2 0 1 0 / 1 1}$ & $\mathbf{2 0 1 1 / 1 2}$ & $\mathbf{2 0 1 2 / 1 3}$ \\
\hline Geral & 7 & 9 & 10 & 10 & 15 & 16 & 46 & 46 \\
Natureza do produto & 0 & 5 & 7 & 8 & 8 & 7 & 28 & 28 \\
Governança corporativa & 36 & 40 & 40 & 40 & 37 & 37 & 55 & 55 \\
Econômico financeira & 16 & 18 & 22 & 18 & 20 & 21 & 28 & 28 \\
Social & 33 & 40 & 37 & 41 & 49 & 51 & 77 & 78 \\
Ambiental & 32 & 36 & 35 & 170 & 168 & 186 & 216 & 216 \\
Ambiental para & 17 & 19 & 22 & 19 & 19 & 19 & 29 & 37 \\
instituições financeiras & & & & & & & & \\
Mudanças climáticas & 0 & 0 & 0 & 0 & 0 & 16 & 19 & 19 \\
Ambiental & 49 & 55 & 57 & 189 & 187 & 221 & 264 & 272 \\
(\% do total geral) & $(35 \%)$ & $(33 \%)$ & $(33 \%)$ & $(62 \%)$ & $(59 \%)$ & $(63 \%)$ & $(53 \%)$ & $(54 \%)$ \\
Total geral & 141 & 167 & 173 & 306 & 316 & 353 & 498 & 507 \\
\hline
\end{tabular}

Fonte: BM\&FBOVESPA (2013b).

- meio ambiente é o conjunto de condições, leis, influências e interações de ordem física, química e biológica, que permite, abriga e rege a vida em todas as suas formas;

- degradação da qualidade ambiental é a alteração adversa das características do meio ambiente;

- poluição é a degradação da qualidade ambiental resultante de atividades que direta ou indiretamente: prejudiquem a saúde, a segurança e o bem-estar da população; criem condições adversas às atividades sociais e econômicas; afetem desfavoravelmente a biota; afetem as condições estéticas ou sanitárias do meio ambiente e lancem matérias ou energia em desacordo com os padrões ambientais estabelecidos;

- poluidor é a pessoa física ou jurídica, de direito público ou privado, responsável, direta ou indiretamente, por atividade causadora de degradação ambiental; e

- recursos ambientais são a atmosfera, as águas interiores, superficiais e subterrâneas, os estuários, o mar territorial, o solo, o subsolo, os elementos da biosfera, a fauna e a flora.

As atividades operacionais das empresas são classificadas em baixo, médio e alto, segundo o potencial poluidor e utilização de recursos naturais, representam uma posição hierárquica do setor em relação à interferência da empresa território (BRASIL, 2000).

O potencial poluidor de uma empresa pode ser definido como qualquer alteração das propriedades físicas, químicas e biológicas do meio ambiente, causada por qualquer forma de matéria ou energia resultante das atividades humanas que, direta ou indiretamente, afetem: a saúde, a segurança e o bem-estar da população; as atividades sociais e econômicas; a biota; as condições estéticas e sanitárias do meio ambiente e/ou a qualidade dos recursos ambientais (BRASIL, 1986).

De acordo com Zamcopé, Ensslin e Ensslin (2012), cada vez mais as empresas têm procurado integrar as variáveis econômicas com as ambientais. Ampliar os impactos classificados pela Lei $\mathrm{n}^{\circ} 10.165$ das empresas integrantes do ISE, pelo cálculo da intensidade vezes escala econômica, amplia a capacidade dos stakeholders avaliarem melhor a sustentabilidade das empresas nas decisões de investimentos.

O uso de métricas que mensurem o uso de recursos naturais e impactos das atividades produtivas, considerando a escala econômica, ampliam os significados tradicionais de ativos e passivos contábeis, relacionando-os à preservação da biosfera, conforme conceitos de equilíbrio e accountability (um dos pilares da governança corporativa: prestação responsável de contas, fundamentação ética e boas práticas contábeis e de auditoria) (KASSAI et al., 2012).

Segundo Conceição et al. (2011), o grau de evidenciação dos parâmetros legais, dentre eles a utilização de recursos naturais e seus impactos, é requisito importante no sentido de manter uma imagem institucional positiva junto ao mercado, conforme apresentado na Tabela 2 , na qual o cumprimento legal representa $16,67 \%$ dos pesos da dimensão ambiental do ISE para o requisito gestão das empresas participantes.

São as empresas que produzem quase tudo que a sociedade consome. A produção pelas corporações gera $60 \%$ do produto interno bruto (PIB) global (IHS, 2014). Assim, o impacto do potencial poluidor e da utilização de recursos naturais depende da intensidade da escala econômica (quantidade produzida e faturamento) das empresas participantes (HAMMOUYA, 1999). 


\section{Método}

Para classificar a dimensão ambiental das empresas integrantes do ISE em 2013 segundo parâmetros da Política Nacional do Meio Ambiente (Lei no 10.165), calculou-se o impacto da escala econômica de cada empresa (intensidade vezes escala econômica). Foram atribuídos pesos $(3=$ baixo, $6=$ médio e $9=$ alto) para as empresas segundo a sua respectiva escala econômica, conforme Tabela 5.

A partir do impacto da escala econômica das empresas do ISE em 2013, ancorado numa avaliação percentil estatística, classificou-se em baixo (B), médio (M) e alto (A) o potencial de poluição e utilização de recursos ambientais das empresas. A escala intervalar baixo, médio e alto foi adotada calculando-se três percentis (percentis são medidas estatísticas separatrizes. Referem-se à posição ocupada por determinada observação numa distribuição. Para obtê-los, os valores da distribuição devem ser ordenados do menor para o maior; em seguida, a distribuição é dividida em partes de modo que cada observação corresponda a um percentil de interesse da distribuição) dos resultados obtidos: até $33 \%$ baixo, de $34 \%$ a $66 \%$ médio e acima de $67 \%$ alto.

Cada empresa classificada (baixo, médio e alto) foi submetida a uma nova classificação, relativa aos percentis dentro de cada intervalo (baixo, médio e alto), a fim de obter a posição relativa, cuja indicação gráfica por setas é apresentada na Tabela 5.

Foram atribuídos pesos (de 1 a 9) para as classificações intervalares relativas das empresas, conforme Tabela 6.

A Tabela 1 apresenta a classificação das empresas integrantes do ISE em 2013, por ordem de precedência (da menor para a maior), pelo impacto da escala

Tabela 5. Indicação de posição intervalar pelo percentil.

$\downarrow$ Até $33 \%$ em cada intervalo baixo, médio e alto

$\rightarrow$ De $33 \%$ a $66 \%$ em cada intervalo baixo, médio e alto

$\uparrow$ Acima de $66 \%$ em cada intervalo baixo, médio e alto

Tabela 6. Peso da classificação intervalar.

\begin{tabular}{cc}
\hline Classificação relativa & Peso \\
\hline $\mathrm{B} \downarrow$ & 1 \\
$\mathrm{~B} \rightarrow$ & 2 \\
$\mathrm{~B} \uparrow$ & 3 \\
$\mathrm{M} \downarrow$ & 4 \\
$\mathrm{M} \rightarrow$ & 5 \\
$\mathrm{M} \uparrow$ & 6 \\
$\mathrm{~A} \downarrow$ & 7 \\
$\mathrm{~A} \rightarrow$ & 8 \\
$\mathrm{~A} \uparrow$ & 9 \\
\hline
\end{tabular}

econômica (intensidade vezes escala econômica), em função do potencial de poluição e utilização de recursos naturais segundo a Lei $\mathrm{n}^{\mathrm{o}} 10.165$.

A escala econômica (R\$ em 2012) foi obtida pela receita líquida das demonstrações financeiras publicadas do exercício de 2012 das empresas integrantes do ISE, a fim de calcular o impacto de cada setor (intensidade vezes escala econômica).

De acordo com Brasil (2007), as empresas listadas na BM\&FBOVESPA são obrigadas a publicar suas demonstrações financeiras no prazo máximo de quatro meses, após o encerramento do exercício social fiscal.

\section{Conclusão e limitações da pesquisa}

O cálculo do impacto (intensidade vezes escala econômica) das empresas integrantes do ISE foi realizado para ampliar a classificação original (baixo, médio e alto) da Lei $n^{\circ} 10.165$, no desafio de os stakeholders avaliarem a sustentabilidade das empresas nas decisões de investimentos. Segundo Meadows (1998), uma das maneiras mais eficientes de alterar o comportamento de um sistema é alterar os indicadores existentes, pois esses podem modificar a perspectiva que se tem sobre a realidade.

A dimensão ambiental do ISE que contempla o potencial de poluição e utilização de recursos naturais representa $62 \%$ do total de pesos para inclusão de uma empresa no índice pelo Conselho Deliberativo, ou seja, a relevância dessa dimensão é inquestionável quando comparada com as demais dimensões avaliadas (Figura 4 e Tabela 4).

Desde a sua criação, em dezembro de 2005 até 2013, o ISE apresentou taxa de crescimento acumulado superior em $85 \%$ ao do Ibovespa (Tabela 3), representando a percepção de valor das empresas do ISE pelos investidores da BM\&FBOVESPA no período, apesar de a metodologia não contemplar a dimensão ambiental pelo impacto da escala econômica das empresas participantes.

Apesar de o desempenho do ISE ser superior ao desempenho do Ibovespa no período de 2006 a 2013, até 2010 o Ibovespa apresentava desempenho superior ao ISE. A percepção de valor do ISE pelos investidores pelo critério taxa de crescimento acumulado ocorreu somente a partir de 2010 (Tabela 3 e Figura 3).

A Tabela 7 agrupa as empresas integrantes do ISE em 2013, segundo classificação de impacto (baixo, médio e alto) da dimensão ambiental pelo impacto da escala econômica (intensidade vezes escala econômica), em função do potencial de poluição e utilização de recursos naturais pelos parâmetros da Lei $\mathrm{n}^{\circ} 10.165$. 
A Tabela 7 apresenta as 11 empresas que exigem maior atenção por parte dos stakeholders (termo usado pela primeira vez pelo filósofo Robert Edward Freeman (FREEMAN; REED, 1984) que se refere a qualquer pessoa ou entidade que afeta, que é afetada ou tem interesse por uma empresa) porque possuem classificação alta do potencial de poluição e utilização de recursos naturais, considerando o impacto ampliado da escala econômica pelos parâmetros da Lei $n^{\circ} 10.165$.

As 6 empresas sem classificação exigem investigação mais profunda por parte dos órgãos legislativos porque não possuem classificação de potencial de poluição e utilização de recursos naturais, podendo confundir os stakeholders que buscam no ISE da BM\&FBOVESPA empresas que se destacam em sustentabilidade a longo prazo.

Considerando que o impacto foi obtido pela intensidade vezes escala econômica, possíveis alterações na escala econômica podem mudar a classificação ampliada da Lei $n^{\circ} 10.165$. Alterações na escala econômica podem ocorrer em função da saída de empresas do ISE e entrada de novas participantes e por outros fatores que alterem a escala econômica.

Outra limitação do método usado é que a Lei $n^{\circ} 10.165$ não oferece subsídios para avaliação dos critérios, científicos ou não, que levaram o legislador a classificar as atividades empresariais em baixo, médio e alto em relação ao potencial de poluição e utilização de recursos naturais, incluindo a não classificação das atividades das empresas BRADESCO, BICBANCO, ITAUUNIBANCO, SANTANDER, SUL AMERICA, BANCO DO BRASIL apresentadas na Tabela 7.

De acordo com Jesinghaus (1999), a principal limitação de um processo complexo de mensuração não se refere apenas como medir, mas como interpretar os indicadores e julgar sua significância para o sistema avaliado (Figura 5 e Tabelas 1, 5, 6 e 7).

Tabela 7. Resumo da classificação de impacto da dimensão ambiental das empresas do ISE em 2013 pelos parâmetros ampliados da Lei $n^{\circ} 10.165$.

\begin{tabular}{clc}
\hline Classificação & \multicolumn{1}{c}{ Empresas } & Quantidade \\
\hline Baixo & ULTRACARGO, EVEN, ECORODOVIAS, ELEKEIROZ, ITAUTEC, AES TIETE, & 11 \\
& CCR, COELCE, CESP, COPASA, OXITENO & 12 \\
Médio & TRACTEBEL, DURATEX, ULTRAGAZ, WEG, ENERGIAS BR, LIGHT, \\
& SUZANO PAPEL, COPEL, FIBRIA, TIM, NATURA, ELETROPAULO \\
Alto & SABESP, CPFL ENERGIA, TELEFONICA BRASIL, CEMIG, OI, BRF FOODS, & \multirow{2}{*}{11} \\
& ELETROBRAS, BRASKEN, GERDAU, IPIRANGA, VALE \\
Sem & BRADESCO, BICBANCO, ITAUUNIBANCO, SANTANDER, SUL AMERICA, \\
& BANCO DO BRASIL & \multirow{2}{*}{6} \\
\hline
\end{tabular}

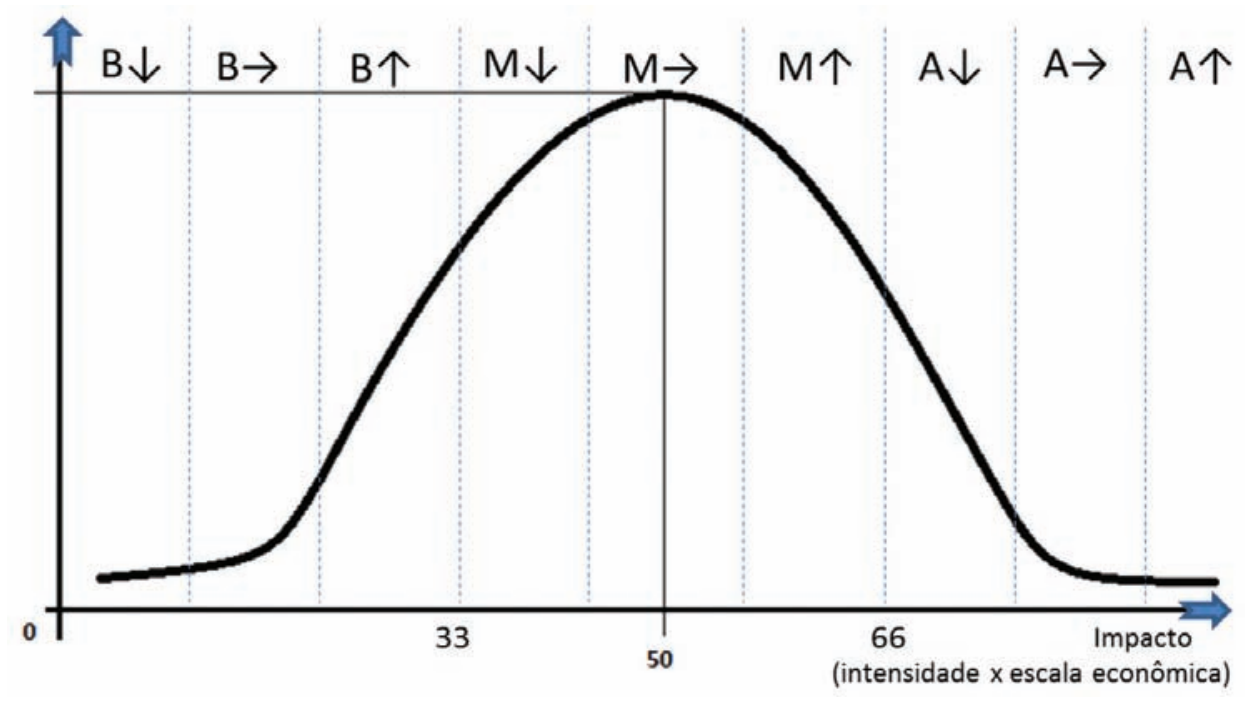

Figura 5. Representação de impacto na curva de Gauss. 


\section{Referências}

ALVES, V. C.; BARBOSA, A. S. Práticas de gestão ambiental das indústrias coureiras de Franca - SP. Revista Gestão \& Produção, v. 20. n. 4, p. 883-898, 2013. http://dx.doi.org/10.1590/S0104-530X2013005000006

BASSETTO, L. I. A incorporação da responsabilidade social e sustentabilidade: um estudo baseado no relatório de gestão 2005 da companhia paranaense de energia - COPEL. Revista Gestão \& Produção, v. 17.n. 3, p. 639-651, 2010. http://dx.doi.org/10.1590/ S0104-530X2010000300016

BERTOLINI, G. R. F; ROJO, C. A.; LEZANA, Á. G. R. Modelo de análise de investimentos para fabricação de produtos ecologicamente corretos. Revista Gestão \& Produção, v. 19. n. 3, p. 575-588, 2012. http://dx.doi. org/10.1590/S0104-530X2012000300010

BM\&FBOVESPA. Índice de Sustentabilidade Empresarial (ISE): composição/carteira do índice. São Paulo, 2012. Disponível em: <http://www.bmfbovespa. com.br/indices/ResumoCarteiraQuadrimestreISE. aspx? ?dioma=pt-br $>$. Acesso em: 21 fev. 2012.

BM\&FBOVESPA. Dimensão ambiental. São Paulo, 2013. Disponível em: <http://www.bmfbovespa.com. br/Indices/download/ISE_Questionario2009.pdf>. Acesso em: 18 out. 2013.

BM\&FBOVESPA. Resumo Taxas médias. 2013a. Disponível em: <http://www.bmfbovespa.com. br/indices/ResumoTaxaMediaCrescimento. aspx ? Indice=ISE\&idioma=pt-br $>$. Acesso em: 29 jul. 2013.

BM\&FBOVESPA. Índices/Resumo Carteira Teórica. 2013b. Disponível em: <http://www. bmfbovespa.com.br/indices/ResumoCarteiraTeorica. aspx ?Indice $=I S E \&$ idioma $=p t-b r>$. Acesso em: 9 maio 2013.

BM\&FBOVESPA. Índices/Resumo Carteira Teórica. 2013c. Disponível em: <http://www. bmfbovespa.com.br/indices/ResumoCarteiraTeorica. aspx ?Indice $=I S E \&$ idioma=pt-br $>$. Acesso em: 9 maio 2013.

BM\&FBOVESPA. Resumo Taxas médias. São Paulo, 2014. Disponível em: <http://www.bmfbovespa. com.br/indices/ResumoTaxaMediaCrescimento. aspx ? Indice=ISE\&idioma=pt-br $>$. Acesso em: 29 jun. 2014.

BRASIL. Conselho Nacional do Meio Ambiente - CONAMA. Resolução Conama nº 001, de 23 de janeiro de 1986. Diário Oficial da União, Poder Executivo, Brasília, DF, 17 fev. 1986.

BRASIL. Lei $\mathrm{n}^{\circ} 10.165$, de 27 de dezembro de 2000. Altera a Lei no 6.938, de 31 de agosto de 1981, que dispõe sobre a Política Nacional do Meio Ambiente, seus fins e mecanismos de formulação e aplicação, e dá outras providências. Diário Oficial da União, Poder Executivo, Brasília, DF, 27 dez. 2000.
BRASIL. Lei $\mathrm{n}^{\circ} 11.638$ de 28 de dezembro de 2007. Altera e revoga dispositivos da Lei $n^{\circ}$ 6.404. Diário Oficial da União, Poder Executivo, Brasília, DF, 28 dez. 2007.

CAMPOS, L. M. S. et al. Relatório de Sustentabilidade: perfil das organizações brasileiras e estrangeiras segundo o padrão Global Reporting Initiative. Revista Gestão \& Produção, v. 20 n. 4, p. 913-926, 2013. http://dx.doi.org/10.1590/S0104-530X2013005000013

CONCEIÇÃO, S. H. et al. Fatores determinantes no disclosure em responsabilidade social corporativa (RSC): um estudo qualitativo e quantitativo com empresas listadas na Bovespa. Revista Gestão \& Produção, v. 18. n. 3, p. 461-472, 2011. http://dx.doi. org/10.1590/S0104-530X2011000300002

DI AGUSTINI, C. A. Mercado de capitais e análise de ações. São Paulo: Globus, 2009.

FERNANDÉS-ARMESTO, F. Os desbravadores. São Paulo: Companhia das Letras, 2009.

FERREIRA, M. E. T. Literatura dos descobrimentos e da expansão portuguesa. Lisboa: Ulisseia, s/d.

FREEMAN, R. E.; REED, D. L. Stockholders and stakeholders: a new perspective on corporate governance. California Management Review, v. 25, p. 88-106, 1984. http://dx.doi.org/10.2307/41165018

HAMMOUYA, M. Statistics on public sector employment: methodology, structures, and trends. Geneva: Bureau of Statistics, International Labour Office, 1999.

IHS. Country \& Industry Forecasting. IHS Global Insight. Disponível em: <www.ihs.com/products/ global-insight/index.aspx>. Acesso em: 9 jun. 2014.

JESINGHAUS, J. Indicators for decision making. Brussels: European Comission, JRC/ISIS/MIA, TP 361, 1-21020, 1999. (Draft Paper).

KASSAI, J. R. et al. Balanço contábil das nações: reflexões sobre os cenários de mudanças climáticas glocais. Brazilian Business Review, v. 9, n. 1. p. 65-109, 2012.

MARCONDES, A. W.; BACARJI, C. D. ISE: sustentabilidade no mercado de capitais. São Paulo: Report, 2010.

MEADOWS, D. Indicators and information systems for sustainable development. Hartland: The Sustainability Institute, 1998.

SHIRREFF, D. Dealing with financial risk. Princeton: Bloomberg Press, 2004.

SILVA, L. S. A.; QUELHAS, O. L. G. Sustentabilidade empresarial e o impacto no custo de capital próprio das empresas de capital aberto. Revista Gestão \& Produção, v. 13. n. 3, p. 385-395, 2006.

ZAMCOPÉ, F. C.; ENSSLIN, L.; ENSSLIN, S. R. Construção de um modelo para avaliação da sustentabilidade corporativa: um estudo de caso na indústria têxtil. Revista Gestão \& Produção, v. 19. n. 2, p. 303-321, 2012. http://dx.doi.org/10.1590/ S0104-530X2012000200006 